\title{
Fracturas orbitarias, lesiones asociadas y predictores de mortalidad en un hospital de IV nivel
}

\author{
Orbital fractures, associated lesions and predictors of mortality in an IV \\ level hospital
}

César Augusto Fernández Dulcey¹, Genny Meléndez Florez ${ }^{2}$, Jesús Ramírez ${ }^{3}$, Silvia Cadena ${ }^{3}$, Diego Camargo ${ }^{4}$, José Suárez ${ }^{4}$

\section{RESUMEN}

Introducción y objetivos. La fractura de órbita constituye una proporción elevada del trauma maxilofacial, que puede resultar en secuelas importantes como amaurosis, diplopía y distopía si el tratamiento es inadecuado o diferido. Nuestra investigación tiene como objetivo determinar la incidencia, lesiones asociadas, el tratamiento y factores de riesgo de mortalidad de las fracturas orbitarias en un hospital de cuarto nivel de complejidad.

Materiales y métodos. Estudio de cohorte prospectivo analítico realizado en una institución nivel IV en el periodo desde 1 febrero de 2016 hasta 31 de enero del 2017. Se estudió las variables sociodemográficas, etiología, tipo de fractura, clasificación orbitarias propuestas por Haug, Nolasco, Zingg, Lang y Dignman, lesiones asociadas, tratamiento quirúrgico y mortalidad.

Resultados. Consultaron 252 pacientes con fractura facial, 105 presentaron diagnóstico de fractura orbitaria. El 92,3\% de los pacientes fueron de género masculino, la edad promedio fue de 36 años. Un $44 \%$ fueron producidos por accidentes de tránsito en moto. La fractura más frecuente fue la derecha. El piso y el reborde orbitario fueron los sitios más comprometidos. Se requirió tratamiento quirúrgico en $63,80 \%$ de las fracturas. Los factores de riesgo independientes de mortalidad fueron el accidente por carro (OR=19,74 IC95\%: 1,41-275,07) y tener alguna complicación (OR=19,74; IC95\%: 3,24163,93)

Conclusiones. La fractura orbitaria tuvo mayor predominancia en hombres jóvenes. Los accidentes de tránsito por moto son una causa importante. La mayoría comprometen el reborde y el piso de órbita. Los factores de riesgo independientes de mortalidad fueron el accidente por carro y tener alguna complicación.

Palabras claves: epidemiología, trauma facial, Colombia, mortalidad fractura, órbita.

\begin{abstract}
Introduction and objectives. Orbital fractures represent a high proportion of all maxillofacial trauma. Fractures of this bone may result in serious complications and sequelae that include amaurosis, diplop and dystopia if treatment is inadequate or differed. This study aims to determine the incidence, related injuries, treatment and mortality predictors of orbital fractures in a fourth level complexity hospital.

Methods. Ananalytic and prospective cohort study developed between February 1, 2016 until January 31 of 2017. Sociodemographic variables, aetiology, fracture type, classifications based on Haug, Nolasco, Zingg, Lang and Dingmann staging systems, related injuries, surgical approaches and mortality.

Results. 252 patients with maxillofacial fractures were included, from which 105 had orbital fractures. $92.3 \%$ were men, the average age was $36.44 \%$ of the fractures were secondary to motorcycle accidents. Right fractures were more frequent. The floor and the orbital rim were the most affected sites. Surgical approaches were needed in $63.80 \%$ of the cases. Associated independent mortality risk factors were: motor vehicle accidents (OR=19.74 $95 \% \mathrm{Cl}: 1.41-275.07)$ and having any type of complication $(\mathrm{OR}=19.74 ; 95 \% \mathrm{Cl}$ 3.24-163.93)

Conclusions. Orbital fractures were most commonly presented in young adults. Motor vehicle accidents are an important cause. The floor and the orbital rim are the most affected sites. Independent mortality risk factors were motor vehicle accidents and having complications.
\end{abstract}

Key words: epidemiology, facial trauma, Colombia, mortality, fracture, orbit.
1. Residente de Cirugía Plástica. Postgrado de Cirugía Plástica Estética y Reconstructiva, Universidad Industrial de Santander

2. Profesora posgrado de Cirugía Plástica. Universidad Industrial de Santander

3. Médica General, Hospital Universitario de Santander, Bucaramanga, Colombia

4. Médica General, Clínica Chicamocha, Bucaramanga, Colombia

$\square$ Correspondencia: Dr. César Fernández Dulcey. Departamento de cirugía plástica estética y reconstructiva. Hospital Universitario de Santander. Сra. 33 \# 28-126 Bucaramanga, Colombia. Tel: +57 3145691778. cesarfernandezdulcey@gmail.com

Los autores no declaran conflictos de intereses.

\section{INTRODUCCIÓN}

El trauma maxilofacial se considera un problema de salud pública, que ocasiona costos elevados al sistema de salud $^{1}$. Las fracturas se clasifican según su localización en fracturas del seno frontal, órbita, malar, arco cigomático, maxilar, Le Fort, nasales, nasoorbitoetmoidales y mandibulares ${ }^{2}$. La fractura de órbita es la quinta fractura más frecuente ${ }^{1}$.

En Brasil se presenta en una relación hombre:mujer que varía en torno de 6,4:1, y las causas más frecuentes son los accidentes en motocicleta, seguidos de los accidentes en carro y la agresión interpersonal ${ }^{3}$. En Colombia existen pocos estudios que describan su incidencia y caracterización ${ }^{4}$.

No se conocen estudios de incidencia de la fractura orbitaria en Santander, Colombia. Por lo tanto, el objeti- 
TABLA 1. Variables sociodemográficas.

\begin{tabular}{|c|c|c|c|}
\hline Variable & $\begin{array}{l}\text { Pacientes con fracturas orbitarias } \\
\qquad \mathrm{n}=105-\mathrm{n}(\%)\end{array}$ & $\begin{array}{l}\text { Pacientes sin fracturas orbitarias } \\
(\mathrm{n}=147)-\mathrm{n}(\%)\end{array}$ & $\mathrm{P}$ \\
\hline Edad media & 24,52 & 29,64 & 0,0006 \\
\hline Masculino & $97(92,38 \%)$ & $125(85 \%)$ & 0,0764 \\
\hline Femenino & $8(7,61 \%)$ & $22(14,96 \%)$ & \\
\hline \multicolumn{4}{|l|}{ Uso de drogas } \\
\hline $\begin{array}{l}\text { Alcohol } \\
\text { Otros tóxicos }\end{array}$ & $\begin{array}{c}24(22,85 \%) \\
4(14,28 \%)\end{array}$ & $\begin{array}{c}35(23,80 \%) \\
14(9,52 \%)\end{array}$ & 0,2554 \\
\hline \multicolumn{4}{|l|}{ Aseguradora } \\
\hline $\begin{array}{l}\text { Seguro automovilístico } \\
\text { Subsidiado } \\
\text { No asegurado } \\
\text { Contributivo } \\
\text { Otros }\end{array}$ & $\begin{array}{c}54(51,42 \%) \\
36(3,4,28 \%) \\
8(7,61 \%) \\
4(3,80 \%) \\
3(2,85 \%)\end{array}$ & $\begin{array}{c}32(21,74 \%) \\
85(57,82 \%) \\
19(12,92 \%) \\
5(3,41 \%)\end{array}$ & 0,0151 \\
\hline \multicolumn{4}{|l|}{ Escolaridad } \\
\hline $\begin{array}{l}\text { Primaria } \\
\text { Secundaria } \\
\text { Otros }\end{array}$ & $\begin{array}{l}40(38,09 \%) \\
25(23,80 \%) \\
40(38,09 \%)\end{array}$ & $\begin{array}{l}51(34,69 \%) \\
45(30,61 \%)\end{array}$ & 0,0959 \\
\hline Procedente de Santander & $89(84,76 \%)$ & $132(89,79 \%)$ & \\
\hline Procedente de Bolívar & $5(4,76 \%)$ & $5(34,01 \%)$ & \\
\hline Procedente de César & $4(3,8 \%)$ & $2(1,36 \%)$ & \\
\hline Procedente de Norte de Santander & $4(3,8 \%)$ & $5(3,40 \%)$ & \\
\hline Sábado & $27(25,71 \%)$ & $23(15,64 \%)$ & 0,0125 \\
\hline Domingo & $24(22,85 \%)$ & $34(23,12 \%)$ & \\
\hline Julio & $14(13,33 \%)$ & $6(4,08 \%)$ & 0,9878 \\
\hline Marzo & $12(11,42 \%)$ & $14(9,52 \%)$ & \\
\hline Diciembre & $11(10,47 \%)$ & $21(14,28 \%)$ & \\
\hline
\end{tabular}

TABLA 2. Incidencia de aparición de las fracturas faciales ( $n=105)$.

\begin{tabular}{|l|l|}
\hline Variable & $\mathrm{n}(\%)$ \\
\hline Tipo de fractura & $66(62,8 \%)$ \\
Simple & $12(11,42)$ \\
Conminuta & $11(10,47)$ \\
Segmentaria & $9(8,57)$ \\
Palo verde & \\
\hline Clasificación de fracturas del techo de órbita & $22(20,92 \%)$ \\
de Haug & $4(3,80 \%)$ \\
No desplazada & $1(0,95 \%)$ \\
Blow-in & $1(0,95 \%)$ \\
Blow-out & $3(2,85 \%)$ \\
Afectación del reborde supraorbitario & $7(6,66 \%)$ \\
Afectación del seno frontal & $6(5,71 \%)$ \\
Combinación & $62(59,04 \%)$ \\
\hline Clasificación de fractura del piso e órbita & $13(12,38 \%)$ \\
de Lang y Dingman & $13(12,38 \%)$ \\
Blow in & $35(33,33 \%)$ \\
\hline Blow-out puro & $19(18,09 \%)$ \\
Blow impuro & $1(0,95 \%)$ \\
\hline Clasificación de Fractura de la pared medial & $7(6,66 \%)$ \\
de la órbita de Nolasco & $1(0,95 \%)$ \\
Tipo I, fractura pura de la pared medial & $10(9,52 \%)$ \\
Tipo Il, fractura de la pared medial y piso. & $13(14,28 \%)$ \\
Tipo III, pared medial, piso y fractura trimalar & $13(12,38 \%)$ \\
Tipo IV, pared medial, piso y fracturas complejas & $13(12,38 \%)$ \\
\hline Clasificación de fractura de pared lateral de órbita & $79,76 \%)$ \\
de Zingg & \\
Tipo A1, fractura aislada del arco cigomático & \\
Tipo A2, fractura aislada de la pared orbital & \\
lateral & $(125,71 \%)$ \\
Tipo A3, fractura aislada del reborde & \\
infraorbitario & \\
Tipo B, fractura tetraploidea & \\
Tipo C, fractura del zigoma multifragmentaria & \\
\hline Combinación de compromiso de reborde orbitario & \\
y alguna pared orbitaria & \\
\hline
\end{tabular}

\section{MATERIALES Y MÉTODOS}

Estudio de cohorte prospectiva analítico. Se compararon los pacientes con trauma facial con diagnóstico de fractura orbitaria y sin fractura de órbita que consultaron al Hospital Universitario de Santander entre el 1 de febrero de 2016 al 1 de febrero del 2017.

Los pacientes elegibles presentaron diagnóstico de fractura facial clínica y tomográfica realizada por un cirujano plástico. Se excluyen los pacientes sin historia clínica. Una vez que los pacientes ingresaron al estudio, se incluyeron los datos sociodemográficos, tiempo de evolución, etiología del trauma, localización y lateralidad de la fractura, lesiones asociadas, tratamiento recibido, complicaciones intrahospitalarias, necesidad de cuidado intensivo (UCI), infección de sitio operatorio (ISO) y muerte. La fractura de órbita se clasificó según las paredes orbitarias afectadas: se usó la clasificación de fractura de techo de la órbita de Haug ${ }^{5}$, de piso de órbita blow-out de Lang ${ }^{6} \mathrm{y}$ blow-in de Dingman ${ }^{7}$, de pared medial de Nolasco ${ }^{8} \mathrm{y}$ de malar de Zingg?.

Se describió el tratamiento quirúrgico recibido, tipo de reducción ósea, maniobras de reducción cerrada, incisiones realizadas, uso de material de osteosíntesis. Este estudio fue aprobado por el comité de ética del Hospital Universitario de Santander. Los datos fueron analizados con el software de análisis estadístico de Stata 13,0 (Stata Corp., College Station, TX). Un análisis descriptivo se realizó de forma inicial. El análisis estadístico fue desarrollado usando el chi-cuadrado y el test de Anova (análisis de va-

vo de nuestro estudio fue determinar la incidencia, las lesiones asociadas y el tratamiento de las fracturas orbitarias en un hospital de cuarto nivel de complejidad durante un año. 
TABLA 3. Signos y síntomas clínicos ( $n=105)$.

\begin{tabular}{|c|c|c|c|}
\hline Signos y síntomas clínicos & $\begin{array}{l}\text { Pacientes con fracturas orbitarias } \\
\qquad n=105-n(\%)\end{array}$ & $\begin{array}{l}\text { Pacientes sin fracturas orbitarias } \\
\qquad(n=147)-n(\%)\end{array}$ & $\mathrm{p}$ \\
\hline Edema & $98(93,33 \%)$ & $138(93,87 \%)$ & 0,8620 \\
\hline Equimosis & $94(89,52 \%)$ & $72(48,97 \%)$ & 0,0003 \\
\hline Dolor & $90(85,71 \%)$ & $132(89,79 \%)$ & 0,0000 \\
\hline Heridas & $57(54,28 \%)$ & $80(54,42 \%)$ & 0,9830 \\
\hline Escalón óseo & $42(40 \%)$ & $36(24,48 \%)$ & 0,0085 \\
\hline Epistaxis & $40(38,09 \%)$ & $65(44,21 \%)$ & 0,3331 \\
\hline Laceraciones & $38(36,19 \%)$ & $41(27,89 \%)$ & 0,1628 \\
\hline Crepitación & $38(36,19 \%)$ & $65(44,21 \%)$ & 0,2028 \\
\hline Hemorragia subconjuntival & $37(35,23 \%)$ & $11(7,48 \%)$ & 0,0000 \\
\hline Depresión palpable & $34(32,38 \%)$ & $17(11,56 \%)$ & 0,0000 \\
\hline Escoriación & $33(31,42 \%)$ & $29(19,72 \%)$ & 0,0336 \\
\hline Hematoma & $24(22,85 \%)$ & $18(12,24 \%)$ & 0,0258 \\
\hline Hipoestesia & $17(16,19 \%)$ & $5(3,40 \%)$ & 0,0004 \\
\hline Compromiso nervio infraorbitario & $14(13,33 \%)$ & $2(1,36 \%)$ & 0,0000 \\
\hline Diplopía & $11(10,47 \%)$ & $2(1,36 \%)$ & 0,0012 \\
\hline Enoftalmos & $10(9,52 \%)$ & 0 & 0,0001 \\
\hline Musculo ocular comprometido & $9(8,57 \%)$ & $1(0,68 \%)$ & 0,0031 \\
\hline Movimientos oculares & $8(7,61 \%)$ & $1(0,68 \%)$ & 0,0033 \\
\hline Anestesia & $6(5,71$ & $1(0,68 \%)$ & 0,0164 \\
\hline Compromiso nervio óptico & $5(4,76)$ & $1(0,68 \%)$ & 0,0094 \\
\hline Atrapamiento recto inferior & $4(3,8 \%)$ & 0 & 0,0031 \\
\hline Atrapamiento recto superior & $4(3,8 \%)$ & 0 & 0,0031 \\
\hline Amaurosis & $4(3,8 \%)$ & $1(0,68 \%)$ & 0,0796 \\
\hline Enfisema & $4(3,8 \%)$ & $1(0,68 \%)$ & 0,0796 \\
\hline Lesión facial & $3(2,85 \%)$ & $5(3,40 \%)$ & 0,8090 \\
\hline Rinoliquia & $2(1,9 \%)$ & $2(1,36 \%)$ & 0,7345 \\
\hline Exoftalmos & $2(1,9 \%)$ & 0 & 0,0937 \\
\hline Telecanto & $1(0,95 \%)$ & $1(0,68 \%)$ & 0,8112 \\
\hline Visión borrosa & $1(0,95 \%)$ & 0 & 0,2375 \\
\hline Compromiso nervio supraorbitario & $1(0,95 \%)$ & $2(1,36 \%)$ & 0,7874 \\
\hline Atrapamiento recto lateral & $1(0,95 \%)$ & $1(0,68 \%)$ & 0,8112 \\
\hline
\end{tabular}

rianza). Valores de $\mathrm{p}<0,05$ fueron considerados significativos. Además se realizó un análisis bivariado y de regresión logística múltiple teniendo en cuenta como desenlace la mortalidad intrahospitalaria. Para la selección de las variables finales, se utilizó la metodología stepwise backward manual, todas las cuales con p menor de 0,05.

\section{RESULTADOS}

Durante un período de 12 meses de nuestro estudio, 31.855 pacientes en total consultaron al Servicio de Urgencias del Hospital Universitario de Santander, de los cuales $0,00791 \%(\mathrm{n}=252)$ fueron por fracturas maxilofaciales, el 0,00329\% $(\mathrm{n}=105)$ correspondían a pacientes con diagnóstico de fractura orbitaria, lo cual da una incidencia de 329 por 100.000 pacientes. El $92,3 \%$ de ellos era de género masculino, con una relación hombre:mujer de 12:1. La edad promedio fue de $36,30 \pm 14,55$ años, con un rango intercuantílico (IQR) de 26 años (Tabla 1).

El tiempo promedio de evolución fue de 24,52 $\pm 27,28$ horas, con un IQR de 17,7 días. En el fin de semana se presentó la mayoría de fracturas, en un 48,56\%. Los meses de julio, marzo y diciembre fueron los de mayor incidencia. El 38,09\% de los pacientes tenía estudios de primaria y 22,85\% ingirió alcohol durante el trauma inicial.

\section{CARACTERIZACIÓN DE LAS FRACTURAS}

En las fracturas orbitarias, la etiología más frecuente fue el accidente en moto, seguida de las lesiones por agresión $(\mathrm{p}=0,8397)$ (Figura 1); el 44,44\% $(\mathrm{n}=49)$ pacientes presentó fracturas orbitarias unilaterales derechas, en el 38,09\% ( $n=40)$ fueron izquierdas y en el 15,23\% $(n=16)$ fueron bilaterales. La pared lateral orbitaria fue las más comprometida, en el $75,2 \%$, seguida de la fractura de piso de la órbita en 59,04\%. El número promedio de sitios fracturados por paciente fue de 2 (Tabla 2). Se tomaron los signos clínicos de todos los pacientes $(n=105)$ y se compararon con los pacientes sin fracturas orbitarias, se evidenció que el edema, equimosis y el dolor fueron los más frecuentes. Signos específicos significativos del trauma orbitario más frecuentes fueron el escalón óseo, la hemorragia subconjuntival y la depresión palpable (Tabla 3).

Se presentó lesiones asociadas con fracturas orbitarias en el 92,38\% ( $n=97)$. Se documentaron Un total de 590 lesiones asociadas, dentro de las cuales la fractura del hueso malar y el trauma craneoencefálico fueron las 2 lesiones asociadas significativas más frecuentes (Tabla 4).

\section{TRATAMIENTO REALIZADO}

Un $63,80 \%(n=67)$ de todos los pacientes con fracturas orbitarias requirieron tratamiento quirúrgico y en $36,19 \%(\mathrm{n}=38)$ se dio manejo conservador. Se eviden- 
TABLA 4. Incidencia de aparición de lesiones asociadas.

\begin{tabular}{|l|c|c|c|}
\hline Lesiones asociadas & $\begin{array}{c}\text { Fractura } \\
\text { orbitaria } \\
(\mathrm{n}=105) \\
\mathrm{n}(\%)\end{array}$ & $\begin{array}{c}\text { Sin fractura } \\
\text { orbitaria } \\
(\mathrm{n}=147) \\
\mathrm{n}(\%)\end{array}$ & $\mathrm{p}$ \\
\hline Fractura de hueso malar & $74(70,47 \%)$ & $12(8,16 \%)$ & 0,0000 \\
\hline Trauma craneoencefálico & $61(58,09 \%)$ & $35(23,80 \%)$ & 0,0000 \\
\hline Fractura de hueso maxilar & $49(46,66 \%)$ & $11(7,48 \%)$ & 0,0000 \\
\hline Fractura de arco cigomático & $49(46,66 \%)$ & $12(8,16 \%)$ & 0,0000 \\
\hline Fractura de hueso nasal & $39(37,14 \%)$ & $73(49,65 \%)$ & 0,0489 \\
\hline Trauma de tórax & $35(33,33 \%)$ & $20(13,60 \%)$ & 0,0002 \\
\hline Trauma de abdomen & $28(26,6 \%)$ & $20(13,60 \%)$ & 0,0091 \\
\hline Hemorragia intracraneana & $27(25,71 \%)$ & $11(7,48 \%)$ & 0,0001 \\
\hline Fractura de hueso frontal & $25(23,80 \%)$ & $9(6,12 \%)$ & 0,0000 \\
\hline Fractura de hueso seno frontal & $22(20,95 \%)$ & $7(4,76 \%)$ & 0,0001 \\
\hline Fractura de hueso mandibular & $19(18,09 \%)$ & $61(41,49 \%)$ & 0,0001 \\
\hline Fractura nasoorbitoetmoidal & $18(17,14 \%)$ & $1(6,80 \%)$ & 0,0000 \\
\hline Herida en cabeza & $17(16,19 \%)$ & $15(10,20 \%)$ & 0,1606 \\
\hline Fractura Le Fort I & $15(14,28 \%)$ & $2(1,36 \%)$ & 0,0000 \\
\hline Herida en extremidades & $14(13,33 \%)$ & $8(5,44 \%)$ & 0,0287 \\
\hline Trauma pulmonar & $13(12,38 \%)$ & $10(6,80 \%)$ & 0,1306 \\
\hline Fractura miembro superior & $9(8,57 \%)$ & $15(10,20 \%)$ & 0,2318 \\
\hline Fractura Le Fort II & $8(7,61 \%)$ & 0 & 0,0006 \\
\hline Fractura de miembro inferior & $6(5,71 \%)$ & $4(2,72 \%)$ & 0,6649 \\
\hline Fractura de hueso palatino & $5(4,76 \%)$ & $6(4,08 \%)$ & 0,7954 \\
\hline Fístula de LCR & $4(3,80 \%)$ & 0 & 0,0154 \\
\hline Fractura de fémur & $3(2,85 \%)$ & $3(2,04 \%)$ & 0,2490 \\
\hline Lesión medular & $3(2,85 \%)$ & $2(1,36 \%)$ & 0,4030 \\
\hline Heridas en tronco & $3(2,85 \%)$ & $3(2,04 \%)$ & 0,6766 \\
\hline Fractura Le Fort III & $2(1,90 \%)$ & $1(0,68 \%)$ & 0,3789 \\
\hline Lesión de plexo braquial & $1(0,95 \%)$ & $2(1,36 \%)$ & 0,7874 \\
\hline Herida vascular & $1(0,95 \%)$ & $1(0,68 \%)$ & 0,8112 \\
\hline Total: & $590 \%$ & 344 & \\
\hline
\end{tabular}

LCR: líquido cefalorraquídeo.

ció que el uso de material de osteosíntesis fue el método preferido de estabilización ósea en el 66\%, se usó de concha auricular en el 15\% de los casos (Figura 2). En los pacientes sin fractura orbitaria se dio tratamiento quirúrgico en $70,67 \%(n=94)$ con $\mathrm{p}=0,631$.

El abordaje quirúrgico más realizado fue el subciliar, seguido de la cola de ceja, los cuales se pueden ver en la

Tabla 5. En cinco pacientes se dio manejo ambulatorio y cien se hospitalizaron. La hospitalización fue en promedio 12,86 $\pm 7,92$ días, con IQR de 11,5 días, siendo mayor en pacientes sin seguro médico (promedio de 16,65 días) y en los que recibieron tratamiento médico de alguna patología médica coexistente (promedio de 16,70 días). La hospitalización en los pacientes sin fracturas orbitarias fue menor (promedio de 8,39 días).

\section{MORBIMORTALIDAD DE LA POBLACIÓN}

La mortalidad del trauma orbitario fue del 4,76\% (Tabla 6), mientras que el 7,61\% requirió UCI; , se presentaron mayores complicaciones en las fracturas orbitarias sin significancia estadística. Los factores de riesgo de mortalidad significativos para los pacientes con fractura orbitaria según la regresión logística fueron el accidente por carro (odds ratio $[\mathrm{OR}]=19,74$; intervalo de confianza 95\% [IC95\%]: 1,41-275,07) y tener alguna complicación médico-quirúrgica $(\mathrm{OR}=19,74$; IC95\%: 3,24-163,93) (Tabla 7).
TABLA 5. Abordajes quirúrgicos ( $n=67)$

\begin{tabular}{|l|c|}
\hline Variable & $\mathbf{n}(\%)$ \\
\hline Abordaje subciliar & $44(65,67 \%)$ \\
\hline Abordaje infraorbitario & $8(11,95 \%)$ \\
\hline Abordaje lateral de ceja & $28(41,79 \%)$ \\
\hline Abordaje directo & $9(13,43 \%)$ \\
\hline Coronal & $1(14,92 \%)$ \\
\hline Vestibular & $5(7,46 \%)$ \\
\hline
\end{tabular}

TABLA 6. Mortalidad y complicaciones de la población.

\begin{tabular}{|l|l|l|l|}
\hline Variable & $\begin{array}{l}\text { Fractura orbitaria } \\
(n=105) n(\%)\end{array}$ & $\begin{array}{l}\text { Sin fractura orbitaria } \\
(n=147) n(\%)\end{array}$ & $P$ \\
\hline Muerte & $5(4,76 \%)$ & $3(2,04 \%)$ & 0,228 \\
\hline Complicaciones & $13(12,38 \%)$ & $8(54,42 \%)$ & 0,7538 \\
\hline UCl & $8(7,61 \%)$ & $3(2,04 \%)$ & 0,032 \\
\hline Neumonía & $3(2,85 \%)$ & $3(2,04 \%)$ & 0,651 \\
\hline ISO & $1(0,95 \%)$ & $2(1,36 \%)$ & 0,798 \\
\hline Meningitis & $1(0,95 \%)$ & 0 & 0,4111 \\
\hline
\end{tabular}

\section{DISCUSIÓN}

La incidencia de fractura orbitaria en el Hospital Universitario de Santander fue de 329 por cada 100.000 habitantes, que comparada con el estudio de Fuertes Valencia en la ciudad de Pasto, evidenció una incidencia de fractura facial de 17 por cada 100.000 habitantes desde el año 2001 hasta $2006^{4}$.

Los hombres presentaron mayor fractura orbitaria, con una relación hombre a mujer de 12:1, una relación mucho mayor que lo publicado por Calderoni, quien encontró un proporción de 6,4:13. La edad promedio fue de $36 \pm 14,55$ años. Varios autores han publicado la edad promedio de presentación entre 31,1 y los 46,8 años ${ }^{10-12}$. Hasta un $22 \%$ consumió alcohol durante el trauma, lo cual coincide con resultados de literatura previos ${ }^{13,14}$.

El fin de semana fueron los días de mayor presentación de fracturas, similar a lo descrito por Ferreira ${ }^{15}$. Los meses de julio, marzo y diciembre presentaron el mayor número de eventos. Hwang encontró resultados concordantes con nuestro estudio ${ }^{10}$.

Los accidentes por motos (44\%) fueron la etiología más frecuente, seguido de la agresión interpersonal en un $22 \%$. Varios autores han encontrado a los accidentes de tránsito como el principal agente etiológico, incluidas las motos ${ }^{3,16}$.

Los síntomas con mayor incidencia fueron el edema y la equimosis, los síntomas específicos más frecuentes fueron el escalón óseo, le hemorragia subconjuntival y la depresión palpable. Calderoni halló resultados similares a los encontrados en nuestro estudio ${ }^{3}$. Chi evidenció en 733 pacientes con fracturas orbitarias blowout, que la equimosis fue el síntoma más frecuente ${ }^{17}$. Bartoli, en un estudio de fracturas de piso de órbita en 213 pacientes desde 2008 al 2013, encontró el signo más frecuente a la hipoestesia del territorio del trigémino seguido de la diplopía ${ }^{18}$.

La hemorragia subconjuntival se presentó como la lesión ocular más frecuente en un 35\%, seguida de enoftalmos, atrapamiento muscular y compromiso del ner- 
TABLA 7. Análisis univariado y regresión logística para mortalidad.

\begin{tabular}{|l|c|c|c|c|c|c|}
\hline & \multicolumn{3}{|c|}{ Análisis univariado } & \multicolumn{3}{c|}{ Regresión logística } \\
\hline & Odds ratio & $P$ & IC95\% & Odds ratio & C & CI 95\% \\
\hline Accidente por carro & 11,47 & 0,045 & $1,05-124,57$ & 19,74 & 0,026 & $1,41-275,07$ \\
\hline Alguna complicación & 23,8 & 0,000 & $4,59-123,27$ & 23,05 & 0,002 & $3,24-163,93$ \\
\hline Fractura Le Fort III & 17,28 & 0,026 & $1,39-213,83$ & 6,96 & 0,340 & $0,129-374,48$ \\
\hline Fractura hueso frontal & 8,70 & 0,014 & $1,53-49,25$ & 1,23 & 0,891 & $0,06-24,96$ \\
\hline Fractura seno frontal & 5,03 & 0,033 & $1,13-22,27$ & 0,99 & 0,995 & $0,09-10,70$ \\
\hline
\end{tabular}

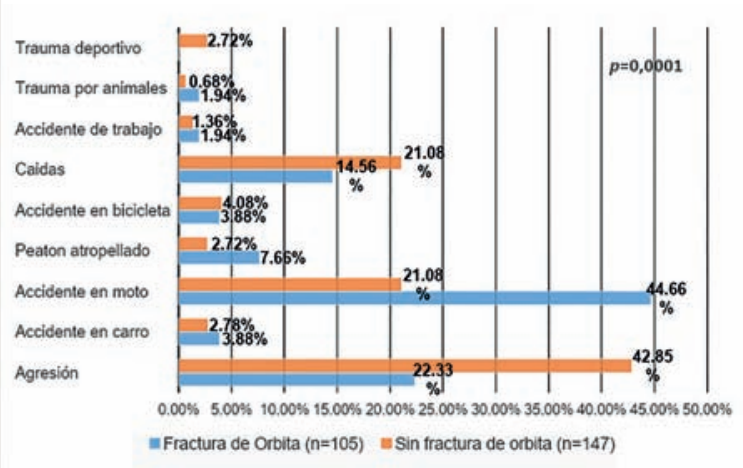

Figura 1. Etiología de las fracturas.

vio óptico. Thurston publicó la tasa de lesión ocular hasta en un $31,8 \%$, siendo mayor en la pared lateral sin significancia estadística ${ }^{19}$. Roh evidenció resultados similares ${ }^{16}$.

La mayoría presentó fracturas orbitarias unilaterales derechas (46,66\%). De Silva encontró compromiso de la hemicara derecha en un $51 \%$, hallazgos similares a los nuestros ${ }^{20}$. La pared orbitaria lateral fue las más comprometida en $75,2 \%$, seguida de la fractura de piso de la órbita en 59,04\%. Rosado observó resultados similares al evidenciar a la fractura del zigoma como la porción orbitaria más afectada, seguido del piso orbitario ${ }^{14}$. Varios autores han encontrado el sitio más comprometido al reborde inferior, lo cual difiere con nuestro estudio ${ }^{3,17}$. La fractura de piso de órbita fue el segundo sitio más frecuente, predominando los blow-out impuros. Roh halló que el 53,74\% son blow impuros, lo cual concuerda con nuestro estudio ${ }^{16}$. El trazo simple fue predominante $(62,8 \%)$, diferente a lo publicado por Chi, quien encontró el trazo conminuto más frecuente $(65,2 \%)$.

Las lesiones asociadas en nuestra población $(92,38 \%)$ fueron mayores que las reportadas en diferentes estudios y diferentes poblaciones, siendo la fractura malar y el trauma craneoencefálico más frecuentes. Tong evidenció fractura del cigoma en 31,2\% y Calderoni el trauma intracraneal en el $17,7 \%$, hallazgos similares a los de nuestro estudio ${ }^{3,21}$. Scolozzi analizó 1433 pacientes con trauma orbitario, evidenciando al enfisema, la diplopía e hipoestesia del infraorbitario como predictores de fractura orbitaria y de manejo quirúrgico $(\mathrm{p}<0,001)^{22}$.

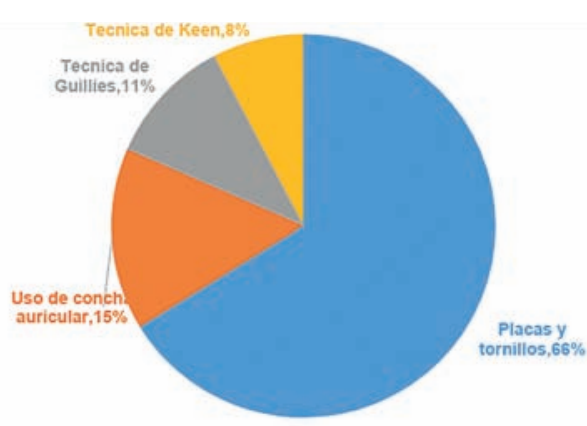

Figura 2. Métodos de tratamiento $(n=67)$.

Se evidenció la hemorragia intracraneana en $25 \%$ de los pacientes. Lee diseñó un estudio retrospectivo de casos y controles con 1220 pacientes, evidenció una tasa del 9\% de lesión intracraneana, hallazgos menores a los nuestros ${ }^{23}$. Otros autores encontraron diferentes asociaciones ${ }^{3,10,17}$.

La estancia hospitalaria en promedio fue de 12,86 días y los días prequirúrgicos en 9,27 días. Hwang et al. hallaron que la duración de estadía fue menor a 1 semana en un $48,3 \%$ de los pacientes, hallazgos menores a nuestros resultados ${ }^{10}$. Ko encontró resultados simila$\mathrm{res}^{24}$. Roh et al. evidenciaron que el $68 \%$ de sus pacientes presentó tiempos entre el trauma y la cirugía menores de 2 semanas $^{16}$.

Se requirió tratamiento quirúrgico y uso de material de osteosíntesis en $63,80 \%$. Varios autores han publicado hallazgos similares en sus investigaciones ${ }^{10.14,16}$. Se usó cartílago de concha auricular en defectos de piso de órbita en $15 \%$, similar a los encontrado por Calderoni $^{3}$. Nowinski describió que el material de osteosíntesis y el hueso costal fue más usado que el cartílago ${ }^{25}$. Se realizó más el abordaje subciliar, en 65,67\%, seguido de la cola de ceja, concordante a lo descrito en el estudio de Calderoni ${ }^{3}$.

La neumonía $(2,85 \%)$ fue la complicación intrahospitalaria más frecuente seguida la ISO (0,95\%). Diferentes autores han reportado incidencia de ISO desde 2,5 hasta 7,8\%3,10. La mortalidad fue del 4,76\%. El accidente por carro (OR=19,74; IC95\%: 1,41-275,07) y tener alguna complicación médica $(\mathrm{OR}=19,74$; IC95\%: 3,24-163,93) fueron factores de riesgo independientes 
y significativos. Ko et al. encontraron una incidencia de mortalidad del 2,38\%, siendo mayor en pacientes hospitalizados que recibieron manejo médico ${ }^{23}$. Diferente de lo encontrado por Bellamy, quien publicó que la fractura Le Fort II está asociada a mortalidad en pacientes con fracturas del tercio medio facial ${ }^{26}$.

Las limitaciones de nuestro estudio fueron que se incluyeron pacientes con manejo ambulatorio que tuvieron estancia hospitalaria corta, además faltó seguimiento a mediano y largo plazo. Se requieren estudios con una mayor población para establecer mayor cantidad de asociaciones entre las lesiones y la mortalidad.

\section{CONCLUSIONES}

La incidencia de trauma orbitario fue elevada, se presentó con alta frecuencia en hombres y jóvenes. Los accidentes en moto fueron una causa importante, se evidenció una alta asociación con fractura malar y trauma craneoencefálico. La mayoría requirió manejo quirúrgico, la mortalidad intrahospitalaria es igual entre pacientes con fracturas orbitarias y sin compromiso orbitario. Los factores de riesgo independientes de mortalidad en pacientes con fractura orbitaria fueron la fractura Le Fort III y el accidente en carro.

\section{BIBLIOGRAFÍA}

1. Allareddy $V$, Allareddy $V$, Nalliah RP. Epidemiology of facial fracture injuries. J Oral Maxillofac Surg 2011 Oct; 69(10):2613-8.

2. Taub PJ, Patel PK, Buchman SR, Cohen M. Ferraro's fundamentals of maxillofacial surgery. New York: Springer;2014.

3. Calderoni DR, Guidi M de C, Kharmandayan P, Nunes PH. Seven-year institutional experience in the surgical treatment of orbito-zygomatic fractures. J Craniomaxillofac Surg. 2011 Dec;39(8):593-9.

4. Fuertes-Valencia L, Mafla ChamorroA, López Ordoñez, E. Análisis epidemiológico de trauma maxilofacial en Nariño, Colombia. CESOdontología 2011; 23(2):33-40.

5. Haug RH, Van Sickels JE, Jenkins WS. Demographics and treatment options for orbital roof fractures. Oral Surg Oral Med Oral Pathol Oral Radiol Endod. 2002 Mar;93(3):238-46.

6. Injuries and diseases of the orbit. Traumatic enophthalmos with retention of perfect acuity of vision. By William Lang, 1889. Adv Ophthalmic Plast Reconstr Surg. 1987;6:3-6

7. Dingman RO, Natvig P. Surgery of Facial Fractures. Philadelphia: WB Saunders; 1964. The Zygoma; pp. 234-5.

8. Nolasco FP, Mathog RH. Medial orbital wall fractures: classification and clinical profile. Otolaryngol Head Neck Surg. 1995 Apr:112(4):549-56.

9. Zingg M, Laedrach $K$, Chen J, Chowdhury $K$, Vuillemin $T$, Sutter $F$, et all. Classification and treatment of zygomatic fractures: a review of 1,025 cases. J Oral Maxillofac Surg. 1992 Aug;50(8):778-90.

10. Hwang K, You SH, Sohn IA. Analysis of orbital bone fractures: a 12year study of 391 patients. J Craniofac Surg. 2009 Jul;20(4):121823.

11. Eom T, Kim Y. Analysis of symptoms according to areas of orbital floor in orbital inferior wall fractures. J Craniofac Surg. 2015 May;26(3):647-9.

12. Scolozzi P, Jacquier P, Courvoisier DS. Can Clinical Findings Predict Orbital Fractures and Treatment Decisions in Patients With Orbital Trauma? Derivation of a Simple Clinical Model. J Craniofac Surg. 2017 Oct;28(7):e661-7.

13. CabalagMS, Wasiak J, Andrew NE, Tang J, KirbyJC, Morgan DJ. Epidemiology and management of maxillofacial fractures in an Australian trauma centre. J Plast Reconstr Aesthet Surg. 2014 Feb;67(2):183-9.
14. Rosado P, de Vicente JC. Retrospective analysis of 314 orbital fractures. Oral Surg Oral Med Oral Pathol Oral Radiol. 2012 Feb;113(2):168-71.

15. Ferreira PC, Amarante JM, Silva PN, Rodrigues JM, Choupina MP, Silva $A C$, et all. Retrospective study of 1251 maxillofacial fractures in children and adolescents. Plast Reconstr Surg. 2005 May; 115(6):1500-8.

16. Roh JH, Jung JW, Chi M. A clinical analysis of bilateral orbital fracture. J Craniofac Surg. 2014 Mar;25(2):388-92.

17. Chi MJ, Ku M, Shin KH, Baek S. An analysis of 733 surgically treated blowout fractures. Ophthalmologica. 2010;224(3):167-75.

18. Bartoli D, Fadda MT1, Battisti A1, Cassoni A1, Pagnoni M1, Riccardi E, et al. Retrospective analysis of 301 patients with orbital floor fracture. J Craniomaxillofac Surg. 2015 Mar;43(2):244-7.

19. Thurston TE, Jackson AS1, Nazir N1, Crowe D, Andrews BT1,2. Risk Assessment of Isolated Single-Wall Orbit Fractures and Eye Injury. J Craniofac Surg. 2018 Jun;29(4):943-945.

20. De Silva DJ, Rose GE. Orbital blowout fractures and race. Ophthalmology. 2011 Aug;118(8):1677-80.

21. Tong L1, Bauer RJ, Buchman SR. A current 10-year retrospective survey of 199 surgically treated orbital floor fractures in a non urban tertiary care center. Plast Reconstr Surg. 2001 Sep 1;108(3):612-21.

22. Scolozzi P1, Jacquier P, Courvoisier DS. Can Clinical Findings Predict Orbital Fractures and Treatment Decisions in Patients With Orbital Trauma? Derivation of a Simple Clinical Model. J Craniofac Surg. 2017 Oct;28(7):e661-7.

23. Lee HJ, Kim YJ, Seo DW, Sohn CH, Ryoo SM, Ahn S, et all. Incidence of intracranial injury in orbital wall fracture patients not classified as traumatic brain injury. Injury. 2018 May;49(5):963-68.

24. Ko MJ1, Morris CK, Kim JW, Lad SP, Arrigo RT, Lad EM. Orbital fractures: national in patient trends and complications. Ophthalmic Plast Reconstr Surg. 2013 Jul-Aug;29(4):298-303.

25. Nowinski D1, Messo E, Hedlund A. Treatment of orbital fractures: evaluation of surgical techniques and materials for reconstruction. J Craniofac Surg. 2010 Jul;21(4):1033-7.Jul;21(4):1033-7.

26. Bellamy JL, Mundinger GS, Reddy SK, Flores JM, Rodriguez ED, Dorafshar AH. Le Fort II fractures are associated with death: a comparison of simple and complex midface fractures. J Oral Maxillofac Surg. 2013 Sep;71(9):1556-62. 